\title{
On some basic considerations of solid/liquid transition models under a microgravity environment ${ }^{1}$
}

\author{
R. Prud'homme ${ }^{2} \&$ M. El Ganaoui ${ }^{3}$
}

\begin{abstract}
Some problems of solid/liquid phase change are presented. Attention is focused on interface modelling for numerical analysis, and one-directional growing. Microgravity relevance of some situations is emphasised. The summary focuses on some activities of the French Research Group: 'Transport Phenomena and Phase Transitions in Microgravity' (GDR-P2TPM) of Centre National d'Etudes Spatiales (CNES)/Centre National de la Recherche Scientifique (CNRS).
\end{abstract}

Keywords: microgravity; solid/liquid transition.

Biographical notes: R. Prud'homme was born in 1938. 1969: PhD Sciences, Univ. Paris. ONERA (1962-69: PhD; 1969-71: Researcher; 1990-2003: Consultant DEFA). CNRS (1971-2004: Researcher; 1992-2003: Director of GDR CNES/CNRS Fluids in Microgravity). Teaching (1972-85: ENSTA; 1978-88: DEA Phys. Liq. Paris VI; 1978-92 Ecole Polytechnique). His field of activities include: relaxing flows; multiphase media; solidification from the melt; plasma jets; interface phenomena; combustion; fluids in microgravity; and critical phenomena.

M. El Ganaoui was born in 1969 in Morocco, 1997: PhD Computational Fluid Dynamics in the University of Aix-Marseille; P. Doc (1997-1998: Researcher IRPHE/CNES); Assistant Professor (1998-1999: University of Avignon); Associate Professor (from 1999: University of Limoges) and Researcher (CNRS Laboratory SPCTS UMR 66 38). His field of activities include: models for problems with solid/liquid transition; CFD/CHT methods and applications to Rayleigh problems; Stefan problem; Marangoni convection; investigation of directional solidification processes in earth and under microgravity conditions; and plasma-surface interaction.

\section{$1 \quad$ Introduction}

Solid/liquid phase changes are sensitive to gravity. Natural convection occurs coupled to solidification front motion. The interfacial zone is often overly complex to describe. Indeed, front curvature and solid phase structure depend on several factors and eliminating effects due to gravity can help us to understand the solidification process.

Experiments under microgravity conditions are performed using drop towers, parabolic flight, sounding rockets and satellites. French Research activity on that field particularly developed in the Group: 'Transport Phenomena and phase transitions in Microgravity' (GDR - P2TPM - CNES/CNRS) is summarised in Prud'homme and Zappoli (2003) and Prud'homme et al. (2001). A topical issue of the Comptes Rendus de l'Académie des Sciences French Journal will also address the state of the art on fundamental subjects in $\mu \mathrm{g}$ environments (Prud'homme et al., 2004). Experiments on transparent material solidification will be performed in DECLIC (Dispositif d'Etude de la Croissance et des Liquides Critiques), which will take place in the ISS (International Space Station) (Prud'homme and Zappoli, 2003). At the same time it is useful to perform calculations with and without gravity effects. Process control by vibration or magnetic field is sometimes required to stabilise solidification fronts.

\footnotetext{
${ }^{1}$ Reference to this paper should be made as follows: Prud'homme, R. and El Ganaoui, M. (2005) 'On some basic considerations of solid/liquid transition models under a microgravity environment', Int. J. Materials and Product Technology, Vol. 22, Nos. 1/2/3, pp. 213-225.

${ }^{2}$ LMM, Université Pierre et Marie Curie/CNRS, 4 Place Jussieu, 75252 Paris Cedex 05, France

${ }^{3}$ SPCTS, Université de Limoges UMR 6638 CNRS, 123 Albert Thomas, 87060 Limoges, France
} 


\section{Basic considerations}

\subsection{Natural convection}

Convection in phase change can be driven by several forces. For typical solidification systems, unless the process takes place under microgravity conditions, on orbiting space platforms, gravity is a present and thermal or compositional inhomogeneities, give rise to density differences. Relying on the mutual orientation of the gravity vector and the density gradient, buoyancy driven convection can be set up significantly altering the thermal and compositional state of the system and affecting the solid/liquid interface shape and mo2tion. This microgravity relevance for fluid flows when density gradients are large and forced convection not too important is generally encountered for Grashov number (Landau and Lifschitz, 1971) of order one or larger:

$$
G r=\Delta \rho g L^{3} / v^{2}
$$

Gr permits us to compare the effects of buoyancy force to viscous drag force. It can be introduced writing the momentum equation as follows:

$$
\rho \frac{d \vec{U}}{d t}+\vec{\nabla} p^{\prime}=\vec{g} \Delta \rho+\mu \vec{\nabla}^{2} \vec{U}
$$

$\Delta \rho$ is the local density deviation (one has $\Delta \rho=\rho-\rho_{\infty}$ ) where $\rho_{\infty}$ corresponds to the liquid at rest at the same altitude), $v=\mu / \rho$ is the kinematical viscosity, $p$ ' is the pressure deviation from the hydrostatic equilibrium, $\vec{U}$ and $\vec{\Gamma}=d \vec{U} / d t$ are local fluid velocity and acceleration respectively. Fluid velocity resulting from buoyancy can be approximated:

$$
\rho U \partial U / \partial x \cong g \Delta \rho \Rightarrow \rho U^{2} / L \cong g \Delta \rho \Rightarrow U \cong \sqrt{L g \Delta \rho / \rho}
$$

The resulting Reynolds number is then:

$$
\operatorname{Re}=U L / v=\sqrt{g L^{3} \Delta \rho / \rho v^{2}}=\sqrt{G r}
$$

The Rayleigh number is more appropriate than the Grashov number in the case of the liquid phase, when coupling with energy equation (Chandrasekhar, 1961) happens and, sometimes, with mass balances of species. Two non-dimensional numbers are then used according to the studied case:

$$
R a_{T}=G r_{T} \operatorname{Pr}=g \beta_{\mathrm{T}} \Delta T L^{3} / v \lambda, R a_{S}=G r_{c} S c g \beta_{\mathrm{S}} \Delta C L^{3} / v D,
$$

where subscripts ( $\mathrm{T}$ ) and (s) refer to thermal and solutal origin of $\Delta \rho: \Delta \rho=\beta_{T} \Delta T$ or $\Delta \rho=\beta_{S} \Delta C$ respectively. Rayleigh numbers take into account the experimental configuration. The classical Rayleigh-Bénard thermal instability occurs in a fluid layer heated from below. The solidification process is generally more complex; for instance, in directional solidification of alloys inside a cylindrical vertical crucible, the solidification layer is moving and the solidification front is not planar, the upward temperature gradient is generally positive, and 
there is a coupling between temperature and concentrations. Radial thermal gradients drive natural convection and modify concentration fields (Benielli et al., 2001). Instability occurs when the appropriate Rayleigh numbers reach critical values corresponding to the considered processes.

\subsection{Flow instabilities and coupling}

In solidification problems, natural convection occurs when the Rayleigh number becomes higher than a critical value which depends in particular on the geometrical configuration. With increasing temperature (or concentration) gradient, higher order critical Rayleigh numbers appear, and result in very complicated situations. At the free surface separating the liquid phase and a surrounding ambient gas, a surface tension caused by thermal and composition inhomogeneities leads to Marangoni convection as occurring in floating zone solidification (or in other solidification procedures, when the contact between the fluid phase and the wall of the crucible is not ensured, as it is often the case in microgravity) (Bennacer et al., 2002; Scriven and Sternling, 1964).

For such problems, phase change interacts with hydrodynamic instabilities, as RayleighBénard or Marangoni-Bénard instabilities (El Ganaoui, 2002; Nield, 1964). This last one can be coupled to the Soret effect, possibly in microgravity (Bergeon et al., 1994; Timchenko et al., 2002). Magnetic fields are used for measuring diffusion coefficients in liquids (Lehmann et al., 1999) or for compensating for gravity, and numerical studies have been performed examining the coupling between buoyancy and magnetic effects (Touhiri et al., 1999).

\subsection{Morphological instabilities}

It has been noted that

"convection effects strongly influence the development of solidification microstructures. However, most microstructure models are based on purely diffusive transport mechanisms. For this reason, crucial benchmark data required to test fundamental theories of microstructural pattern formation have resulted from solidification experiments conducted in microgravity environment, where convection effects can be substantially reduced, and/or from ground-based experiments with restricted samples sizes where convection effects can be eliminated" (Karma, 2001)

With this in mind, the conditions for instabilities to appear in directional solidification of dilute alloys were given by Mullins and Sekerka (1964).

In the case of binary lamellar eutectics, relations between lamellar characteristic spacing, temperature gradient and front velocity was found first by Jackson and Hunt (1965), who also gave a first estimate of the stability bounds of the lamellar pattern.

These theories apply to highly idealised systems (Langer, 1986). They cannot explain complex phenomena observed in real systems such as curvature of the front in relation to convective instabilities (Figure 1), or eutectic colony formation. These last phenomena have been observed in thin-sample directional solidification of the transparent eutectic alloy $\mathrm{CBr}_{4}-$ $\mathrm{C}_{2} \mathrm{Cl}_{6}$ showing the existence of colonies when impurities are present (Akamatsu and Faivre, 2000). The transition between planar lamellar eutectic front and eutectic colonies goes through intermediate structures as travelling waves and two-phase fingers which have recently been observed (Akamatsu and Faivre, 2000) and are now studied by linearised theory or by the phase field method (see Section Phase field model for microstructures). 


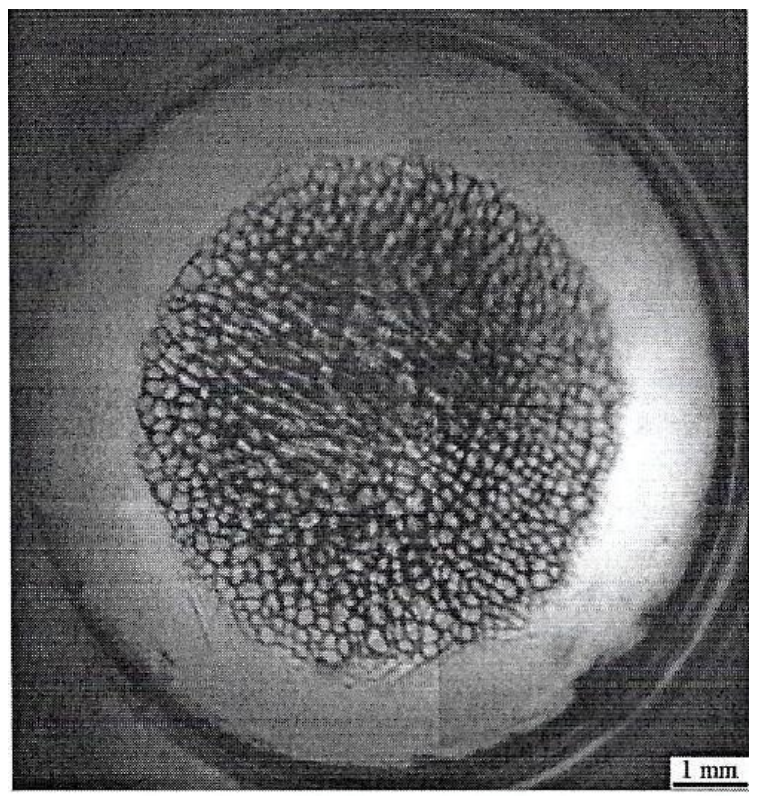

Figure 1 Interface observation from the top in growth direction for a thick solid seed: cells are localised in the middle of the interface (SCN-0.2\%Acetone, $\mathrm{V}=1.4 \mathrm{pm} / \mathrm{s})($ Benielli et al., 2001)

\section{Interfacial modelling and applications}

\subsection{Solid/liquid transition zone}

The solid/liquid transition zone is often very complex to study. In some cases of directional solidification in alloys, the solidification front appears as a smooth (but generally nonplanar) surface with local thermochemical equilibrium between solid and liquid. In other cases, for sufficiently high values of pulling velocity, microstructures resulting from an instability process appear, but the interface remains relatively smooth. In the case of lamellar eutectic alloys it is important to determine the size and distribution of microstructures in relation to the external constraints. The interfacial morphology becomes very complicated for dendrites and for dendritic layers.

Local balance equations resulting from irreversible thermodynamics can be written in each case, both for the interface and for the bulk phases. A critical problem for numerical resolution is the presence of an interfacial discontinuity at the boundary between phases. To avoid this difficulty, some authors have replaced the discontinuity by a continuous description of the transition region. This is the concept of phase field. Two classes of phase field method, which differ by the scale of the description (macroscopic, and microscopic - or mesoscopic, but not molecular) are presented here.

One of the macroscopic methods is applied to dendritic-like porous structures. The main difference with the other cases is $t$ a 'mushy zone' between fully solid and liquid phases is considered (Camel and Arquis, 1992; Goyeau et al., 1999). In this method of prescribing a Darcy source term the velocity value arising from the solution of the momentum equation are inhibited, reaching values close to zero on complete solid formation. The Darcy source term $-\frac{\mu_{1}}{K} \frac{\rho}{\rho_{1}}\left(\vec{v}-\vec{v}_{S}\right)$ appears on the right-hand side of momentum equation where $K$ denotes the isotropic permeability and represents the direct link between microscopic morphology and the macroscopic equations of conservation. Here, $K$ is a scalar for simplicity (Akamatsu and 
Faivre, 2000; Benielli et al., 2001). It is expressed as a function of the volume fraction $f_{l}$, of the liquid. In a more general case it becomes a second order tensor. Closure relations were given (Favier and Camel, 1986; Goyeau et al., 1999) for the isotropic case, and more recently for the non isotropic one.

In the following, basic equations of a macroscopic model, which is called the enthalpy method, and some results of its application to particular cases, are given. Then the phase field model for microstructures is briefly presented. The two methods give continuous balance equations that are valid in the whole considered volume, containing solid and fluid phases.

\subsection{Enthalpy method for homogeneous macroscopic approach}

The enthalpy approach for directional solidification (Benielli et al., 2001; El Ganaoui, 2002; El Ganaoui et al., 2002; Guérin et al., 2001) considers a single set of homogenous balance equations adapted to the problem of phase change in a 2D configuration:

$$
\begin{gathered}
\frac{\partial \rho}{\partial t}+\vec{\nabla} \cdot(\rho \vec{v})=0 \\
\frac{\partial \rho C}{\partial t}+\vec{\nabla} \cdot(\rho \vec{v} C)=\vec{\nabla} \cdot\left(\rho D_{l} C_{l}\right)+\vec{\nabla} \cdot\left(\rho\left(C_{l}-C\right)\left(\vec{v}-\vec{v}_{S}\right)\right) \\
\frac{\partial \rho u}{\partial t}+\vec{\nabla} \cdot(\rho \vec{v} u)=-\frac{\partial p}{\partial x}+\nabla \cdot\left(\mu_{l} \frac{\rho}{\rho_{l}} \vec{\nabla} u\right)-\frac{\mu_{l}}{K} \frac{\rho}{\rho_{l}}\left(u-u_{S}\right) \\
\frac{\partial \rho v}{\partial t}+\vec{\nabla} \cdot(\rho \vec{v} v)=-\frac{\partial p}{\partial y}+\nabla \cdot\left(\mu_{l} \frac{\rho}{\rho_{l}} \vec{\nabla} v\right)-\frac{\mu_{l}}{K} \frac{\rho}{\rho_{l}}\left(v-v_{S}\right)+\rho_{0} g \beta_{T}\left(T-T_{F}\right) \\
\frac{\rho h}{\partial t}+\vec{\nabla} \cdot(\rho \vec{v} h)=\vec{\nabla} \cdot\left(\frac{k}{c} \vec{\nabla} h\right)+\vec{\nabla} \cdot\left(\frac{k}{c} \vec{\nabla}\left(h_{S}-h\right)\right)+\vec{\nabla} \cdot \rho\left(h_{l}-h\right)\left(\vec{v}-\vec{v}_{S}\right)
\end{gathered}
$$

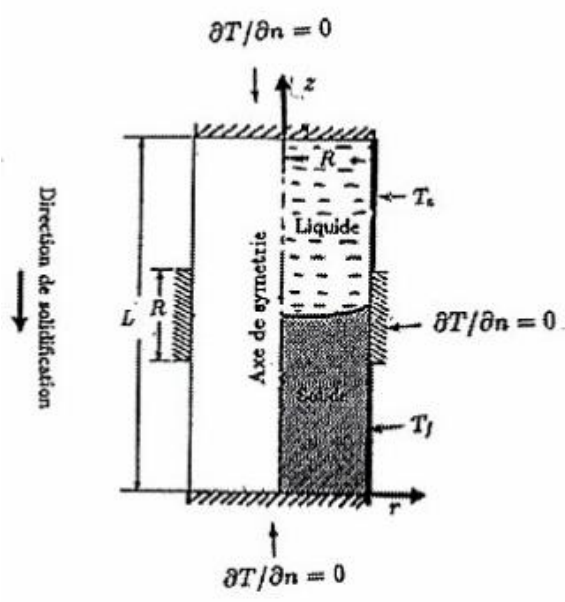

a
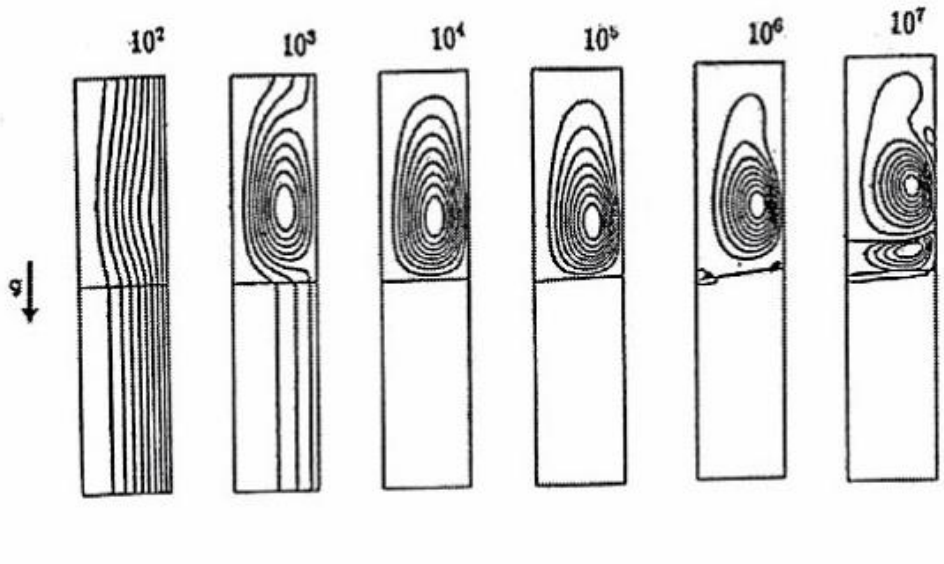

b

Figure 2 Illustration of the enthalpy-porosity based computations: melt under directional solidification using vertical Bridgman method (a) for $10^{2} \leq \mathrm{Ra}_{\mathrm{T}}=g \beta\left(t h-T_{c}\right), L^{3} / v \alpha \leq 10^{-7}$ (i.e. gravity effect) showing evolution from monocellular to multicellular flow (b). Simulation corresponding to an enthalpy porosity formulation/Finite Volumes method (El Ganaoui, 2002) 
where $\rho$ is an average density weighted by the liquid and solid mass fractions, $\vec{v}, \vec{v}_{S}$ and $C$ and $h$ are averaged local quantities weighted by the liquid and solid volume fractions. For instance one has $C=f_{l} C_{l}+\left(1-f_{l}\right) C_{S}$.

With this model, including appropriate closure relations and boundary and initial conditions, it is possible to obtain isotherms streamlines which show in particular, the position of a curved smooth interface. The numerical solution is obtained by the enthalpy model associated with a finite volume approximation. One obtains evolutions of the melt-solid interface, and of velocity and solutal fields. An illustration of flow evolving from monocellular to multicellular convective cells with thermal $\mathrm{Ra}$ is given in Figure 2 and coupling between buoyancy and magnetic effects in Figure 3.
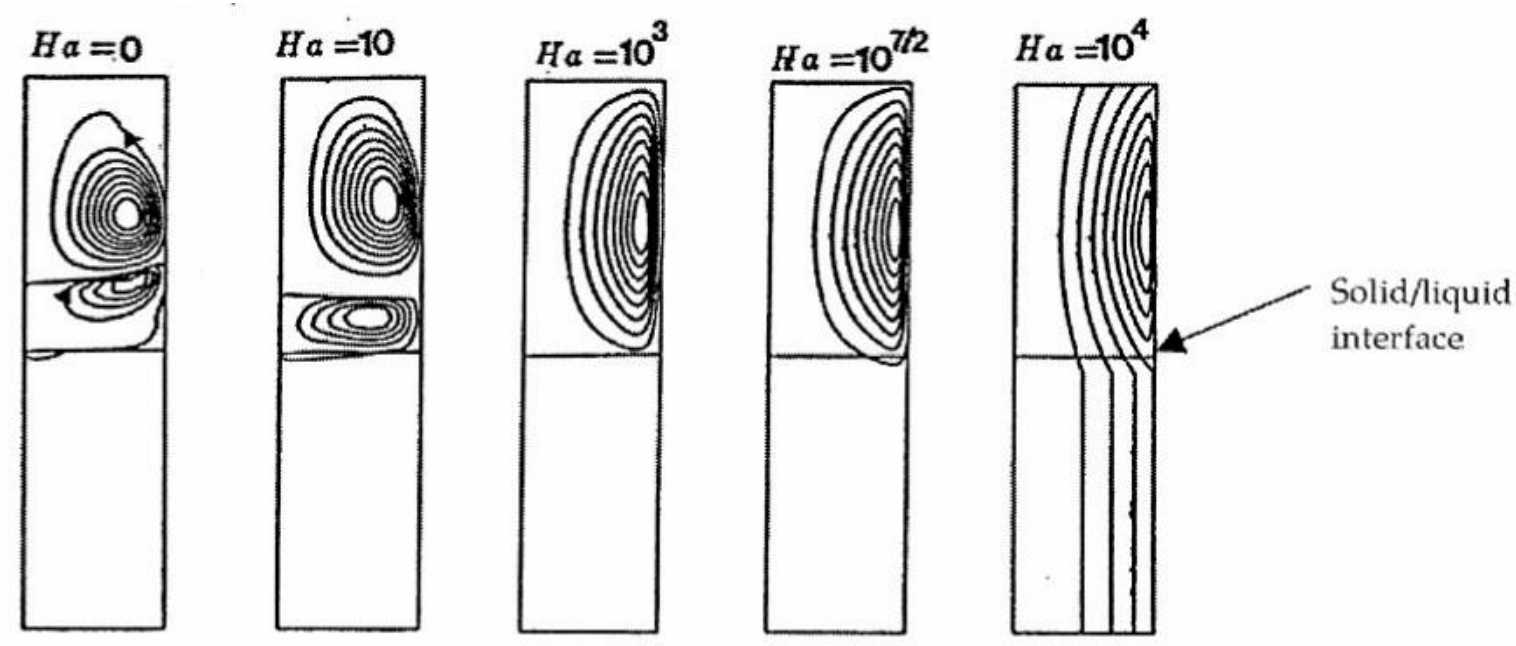

Figure 3 Illustration of the enthalpy-porosity based computations: effect of constant magnetic field $\left(0 \leq H a=\left(\sigma B L^{2} / \mu\right)^{1 / 2} \leq 10^{4}\right)$ opposite to gravity on multi-cellular flow corresponding to $\mathrm{Ra}=10$ for vertical Bridgman configuration (El Ganaoui, 2002)

Figure 4 is obtained in a case where the porous term is not relevant (Benielli et al., 2001). The position of numerical isotherms is in good agreement with front recoil measurements performed on pure succinonitrile (SCN). This result gives information on the contribution of thermal convection to this recoil. However, the authors point out the discrepancy between a purely diffusive model and experimental data. Finally, it appears that comparative studies with and without convection are required to clarify and quantify convection influence, therefore underlining the great interest of microgravity (DECLIC spatial project) for solidification studies. 

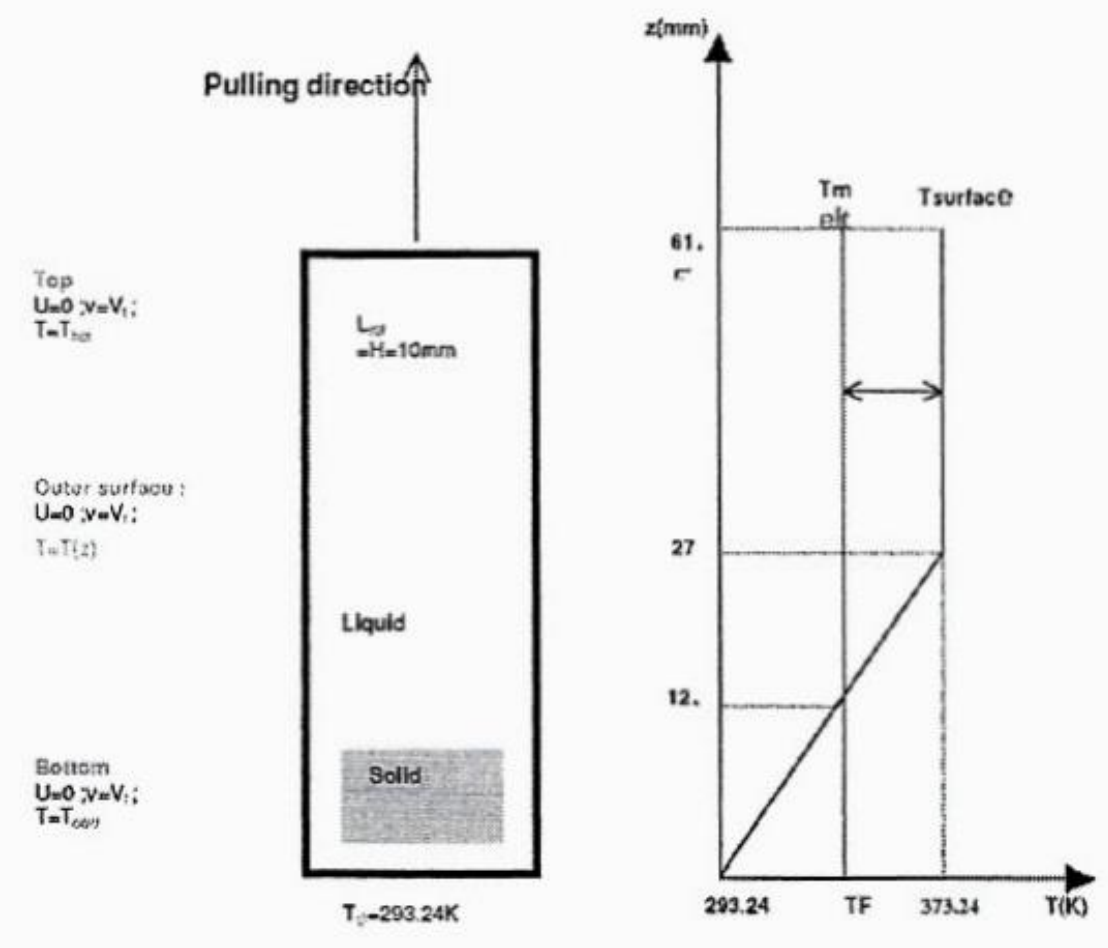

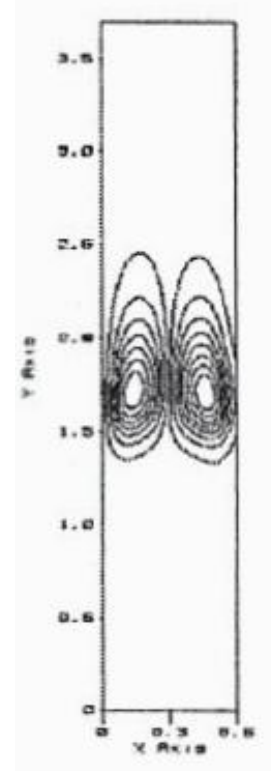

a

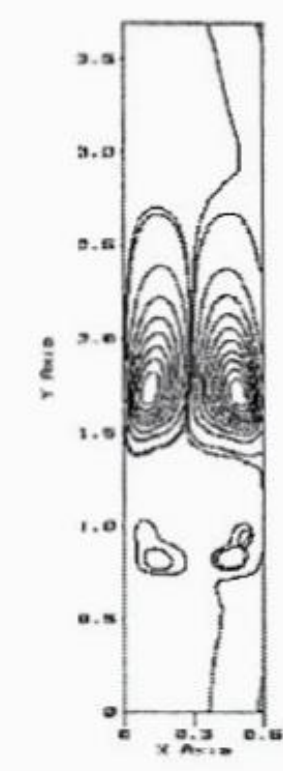

b

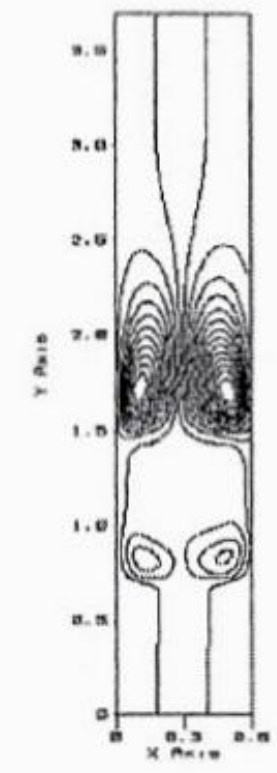

c

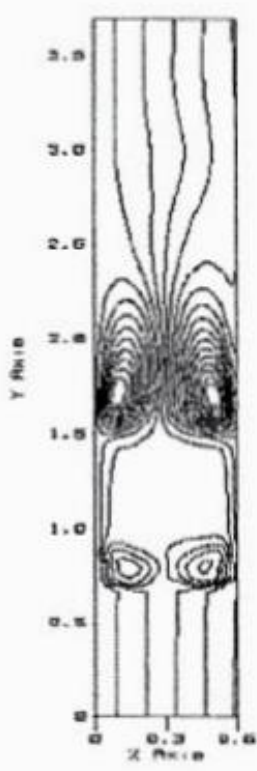

d

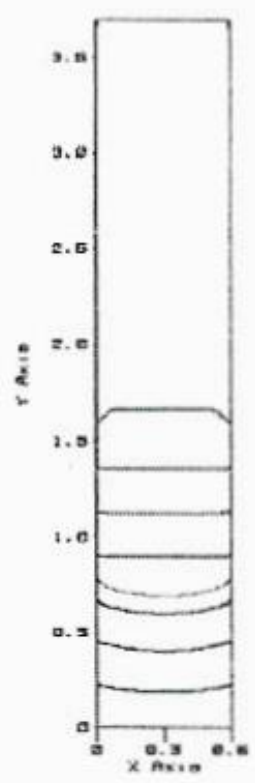

e

Figure 4 Illustration of the enthalpy-porosity based computations for experiments: simplified sketch of SNC growth configuration (top), the ampoule is $61 \mathrm{~mm}$ higher and $10 \mathrm{~mm}$ larger submitted to a thermal gradient of 30 $\mathrm{K} / \mathrm{cm}$. Bottom: stream plots obtained for various pulling velocities (a) $V_{t}=0 \mu \mathrm{ms}^{-1}$, (b) $V_{t}=1 \mu \mathrm{ms}^{-1}$, (e) $V_{t}=5$ $\mu \mathrm{ms}^{-1}$, (d) $V_{t}=10 \mu \mathrm{ms}^{-1}$, (e) isotherms for $V_{t}=10 \mu \mathrm{ms}^{-1}$, interface position around $\mathrm{y}=0.75$ corresponds to the grey line (Benielli et al., 200!). Corresponding control parameters are Rayleigh number $R a=\frac{g \beta\left(T_{h}-T_{c}\right) L^{3}}{v \alpha}=1.110^{6}$, Prandtl number $\operatorname{Pr}=\frac{v}{\alpha}=22.8$ and Stefan number Ste $=$ $\frac{C_{s}\left(T_{h}-T_{c}\right)}{L_{f}}=1,71$ 


\subsection{Phase field model for microstructures}

To explain microstructures in constrained growth, and recently in eutectic colony formation (Akamatsu and Faivre, 2000), Plapp and Karma (1999) have studied the problem first analytically, and then numerically, using a phase field model (Plapp and Karma, 2001). They studied a ternary alloy close to a binary eutectic which, for simplicity, assuming a symmetric phase diagram. They introduced a phase field $\phi(x, z, t) \mathrm{z}, \mathrm{t})$ that distinguishes between solid and liquid, i.e. a scalar field that has a fixed value in the bulk phases and varies continuously across the smooth, diffuse interface. They started from a dimensionless Helmholtz free energy functional, and they deduced, by minimisation, three local equations between three variables, which are valid everywhere under appropriate assumptions:

$$
\begin{aligned}
\tau \frac{\partial \phi}{\partial t} & =W_{\phi} \vec{\nabla}^{2} \phi+\frac{\phi}{2}-\frac{\phi^{3}}{2}+h^{\prime}(\phi)\left(f_{\text {liq }}-f_{\text {sol }}\right) \\
\frac{\partial u}{\partial t} & =\vec{\nabla} \cdot\left[M\left(\frac{\partial^{2} f_{l i q}}{\partial u^{2}}+W_{u}^{2} \vec{\nabla}\left(\vec{\nabla}^{2} u\right)\right)\right] \\
\frac{\partial C}{\partial t} & =\vec{\nabla} \cdot\left(\bar{M} \frac{\partial^{2} f_{l i q}}{\partial \bar{C}^{2}} \vec{\nabla} C\right)
\end{aligned}
$$

In these equations, $u$ is a scaled concentration $u(x, z, t)=\frac{C(x, z, t)-C_{E}}{\left(C_{\beta}-C_{\alpha}\right) / 2}$ where $C_{E}, C_{\alpha}$, and $C_{\beta}$ are the compositions of the liquid and the two solid phases in the pure binary eutectic at the eutectic temperature $T_{E}, \tilde{C}(x, z, t)$ is the concentration of impurities, $h(\phi)=\frac{3}{2}\left(\phi-\frac{\phi^{3}}{3}\right)$ is an interpolation function, $f_{\text {liq }}(u, \tilde{C}, T)$ and $f_{\text {sol }}(u, \tilde{C}, T)$ are the free energy densities for liquid and solid, respectively, $W_{u}$ and $W_{\varphi}$ are characteristic thicknesses of the solid-solid and of the solidliquid interfaces, respectively, and $M(\phi, u, \tilde{C})$ and $\tilde{M}(\phi, u, \tilde{C})$ are the mobility functions of the eutectic component and the ternary eutectic, respectively.

Numerical simulations which have been performed with this phase field model, have enabled the authors to carry out large-scale simulations of colony formation starting from arrays of up to 40 lamella pairs. They found a good overall agreement with the theoretical predictions of linear analysis, predicting a destabilisation front by long-wavelength modes that may be stationary or oscillatory. However, they found that the growth of the lamella is not exactly normal to the large-scale envelope of the composite interface, a rule used in the linear stability analysis (Plapp and Karma, 1999).

The phase field model must be extended to 2D interfaces in 3D configuration (Dejmeck et al., 2001). Akamatsu et al. (2001) compare results obtained in thin and massive sample experiments. In the first case, one generally has a flat solid-melt interface. In the last case, the 2D solidification front presents a curvature because the isotherms are curved for well known technical reasons. This curvature is, of course, influenced by gravity but it also exists in microgravity. Thermosolutal convection will also lead to modifications in local dynamics of eutectic fronts and can also cause a macro-segregation. Other specific phenomena are pointed out for 2D fronts: varying orientations of lamella between eutectic grains, global slow rotation 
of the microstructure around the $\mathrm{z}$ axis, presence of 'faults'. The authors propose to compare benchmark space experiments to ground ones, and also to study the intermediate case of semimassive samples.

\subsection{Second gradient for liquid-liquid interaction}

Some fluid-fluid interfaces have been studied by second gradient methods (Casal, 1972; Seppecher, 1987). In the second gradient model, internal energy per unit mass depends not only on the entropy and the density but also on the magnitude of the density gradient (Gatignol and Prud'homme, 2001):

$$
e=e\left(s, \rho,|\vec{\nabla} \rho|^{2}\right)
$$

The associated Gibbs relation is:

$$
d e=T d s-p d(1 / \rho)+(\lambda / \rho) \vec{\nabla} \rho \cdot d(\vec{\nabla} \rho)
$$

where $\lambda$ is a coefficient called the 'capillarity coefficient'. Using average quantities and introducing the volumetric masses of the species and their gradients, this type of model can certainly be extended to solidification fronts in mixtures and can be considered as a phase field model (Gatignol and Prud'homme, 2001; Gouin, 1998).

\section{Other relevant solidification aspects}

To complement previous sections and conclude this summary, some solidification problems relevant for microgravity experiments and comparison with numerical results are quoted.

Melting and thermal stabilisation stages preparatory to directional solidification greatly affect the initial state on which growth is started (Benielli et al., 2002), and then directional solidification (Akamatsu and Faivre, 2000). It is pointed out that free melting is not the mere reversal of free crystal growth. Two phenomena have been observed in situ and in real time during directional melting of transparent succinonitrile-acetone alloys in a cylinder: nucleation and growth of liquid droplets in the bulk solid and liquid dendrites, due to morphological instability of the phase boundary. Benielli et al. (2001, 2002) analysed the dynamics of melting morphology at the solid-liquid interface. The criterion for morphological instability in directional melting reads:

$$
V>V_{M I}=\left(k D_{s}+D_{l}\right) G /\left[m C_{0}(k-1)\right]
$$

with $V_{M I}$ the critical melting velocity, $C_{0}$ the initial solute concentration. The authors emphasise the interest in carrying out experiments to the limit of the diffusive transport of solute in the liquid that works in the absence of gravity. They participate in the DECLIC project of CNES (Prud'homme and Zappoli, 2003).

Berne et al. (2001) studied crystalline nucleation and phase selection in refractory metals and alloys in the 50-m high Grenoble drop-tube of CEA. They emphasise the interest of microgravity and ultra-vacuum to ensure absolute no-contact conditions and purification by evaporation of spherical droplets. 
Camel and Dupouy (2001) study the influence of natural convection on the formation of dendritic microstructures in the columnar/equiaxed transition range in the specific case of Al$3.5 \mathrm{wt} \% \mathrm{Ni}$ alloy.

Nguyen et al. (2001) stress the importance of rnicrostructures for the resulting properties of materials. Spatial experiments $L M S$ and STS-95 on directional solidification of Al-3.5 wt\% Ni alloy enabled them to understand the role played by preparatory phases of melting and stabilisation, and the initial directional-solidification transient, so that the measured concentration variation in solidified samples in the AGHF (Advance Gradient Heating Facility) can be explained.

The problem of solidification front curvature is very important and experiments will be performed on transparent material for a better know of these phenomena in the DECLIC instrument.

Process control by vibration was studied for stabilising solidification fronts and obtaining planar fronts. Reduced gravity experiments are to be performed in this field, for a better understanding of the effects of vibrating fields on fluids, interfaces and multiphase flows (Lyubimov et al., 1997).

Fina it should be borne in mmd that, even in microgravity conditions, solidification remains sensitive to the gravity level and orientation as shown by Simpson and Garimella (2000).

\section{Acknowledgements}

The authors acknowledge the researchers of the GDR group, and also D. Gobin and M. Plapp, for communicating their interesting papers to make possible this review on French research in a $\mu \mathrm{g}$ context.

\section{References}

Akamatsu, S. and Faivre, G. (2000) 'Traveling waves, two-phase fingers, and eutectic colonies in thin-sample directional solidification of a ternary eutectic alloy', Physical Review E (Statistical Physics, Plasmas, Fluids, and Related Interdisciplinary Topics), Vol. 61, pp.3757-3769.

Akamatsu, S., Bottin-Rousseau, S. and Faivre, G. (2001) 'La dynamique de solidification des eutectiques lamellaires: des échantillons minces aux systèmes massifs', J. Phys. 1V France, Vol. 11, pp.127-134.

Benielli, D., El Ganaoui, M., Semma, E., Bergeon, N., Jamgotchian, H., Voge, P., Billia, B. and Bontoux, P. (2001) Effect of the thermal convection on directional solidification of succinonitrileacetone alloy: Comparison between experimental and numerical studies', J. Phys. IV France, Vol. 11, pp.135-142.

Benielli, D., Bergeon, N., Jamgotchian, H., Billia, B. and Voge, Ph. (2002) 'Free growth and instability morphologies in directional melting of alloys', Phys. Rev. E, Vol. 65, 051604.

Bennacer, R., El Ganaoui, M., Mohammad, A.A. and Fauchais P. (2002) 'Computational analysis of Marangoni effects during floating zone growth under micro-gravity conditions', in S. Dost, H. Struchrup and I. Dincer (Eds) Progress in Transport Phenomena, Paris: Elsevier, pp.559-564.

Bergeon, A., Henry, D. and Benhadid, H. (1994) 'Marangoni-Bénard instability in microgravity conditions with Soret effect', International Journal of Heat and Mass Transfer, Vol. 37, p. 1545

Berne, C., Pasturel, A. and Vinet B. (2001) 'Germination et sélection de phases dans les métaux et alliages réfractaires: approches expérimentales en tube à chute libre et théorique par calculs $a b$ initio', J. Phys. IV France, Vol. 11, pp. 179-186. 
Camel, D. and Arquis, E. (1992) Structural effects of convective flows in dendritic solidification numerical modelling, EUROMECH 284 on Fluid Flow phenomena in Crystal Growth, Aussois, 13-16 October.

Camel, D. and Dupouy, MD. (2001) 'Comparative study of the columnar-equiaxed transition in microgravity and on ground during directional solidification of a refined Al-3.5 wt\% Ni alloy', $J$. Phys. IV France, Vol. 11, pp. 119-126.

Casal, P. (1972) 'La théorie du second gradient et la capillarité', C. R. Acad. Sci. Paris, Vol. 274, Série A, pp. 1571-1574.

Chandrasekhar. S. (1961) Hydrodynamic and Hydro-magnetic Stability, Oxford: Clarendon Press.

Dejmeck, M., Fleury, V., and Plapp. M. (2001) 'Etude numérique de la croissance dendritique et cellulaire', Colloque 'Sciences de la matière et microgravité', 14-15 Mai, ESPCI. Paris.

El Ganaoui, M. (2002) 'Computational modeling of heat mass and solute transport in solid/ liquid transition systems on earth and on microgravity environment', in S.R. Idelsohn, V. Sonzogi and A. Cardona (Eds.) Mecanica Computational Vol. XXI, pp. 2587-2592.

El Ganaoui, M., Lamazouade, A., Bontoux, P. and Morvan, D. (2002) 'Computational solution for fluid flow under solid/liquid phase change conditions', Int. J. Computers and Fluids, Vol. 31, Nos. 47, pp. 539-556.

Favier, J.J. and Camel, D. (1986) 'Analytical and experimental study of transport processes during directional solidification and crystal growth', J. Crystal Growth, Vol. 79, pp. 50-62.

Gatignol, R. and Prud'homme, R. (2001) 'Mechanical and thermodynamical modeling of fluid interfaces', Series on Advances in Mathematics for Applied Sciences, Vol. 58, World Scientific.

Gouin, H. (1998) 'Energy of interaction between solid surfaces and liquids', J. Phys. Chem. B, Vol. 102, pp. 1212-1218.

Goyeau, B., Benihaddadene, T., Gobin, D. and Quintard, M. (1999) 'Numerical calculation of the permeability in a dendritic mushy zone', Metallurgical and Materials Transactions, Vol. 30B, pp. 613-622.

Guérin, R., El Ganaoui, M., Haldenwang, P. and Bontoux, p. (2001) 'Finite volume and spectral methods applied to Pb-30\%Tl alloy solidification', J. Phys. IV France, Vol. 11, pp. 151-159

Jackson, K.A. and Hunt, J.D. (1965) Acta Metall., Vol. 13, pp. 1212-1215.

Karma, A. (2001) 'Microstructural pattern formation during solidification', Introduction à la session 2 (Solidification et croissance cristalline) du Colloque 'Sciences de la matière et microgravité', 14-15 Mai, ESPCI, Paris.

Landau, L. and Lifschitz, E. (1971) Mécanique des Fluides, Editions MIR.

Langer, J.S. (1986) 'Recent developments in the theory of pattern formation', Physica A:

Statistical and Theoretical Physics, Vol. 140, Nos, 1-2, pp. 44-50.

Lehmann, P., Alboussière, T., Moreau, R. and Uspenski, V. (1999) 'MMD convection control applied to chemical diffusivities measurements', J. Chim. Phys., Vol. 96, pp. 1 105-1110.

Lyubimov, D.V., Cherepanov, A.A., Lyubimova, T.P, and Roux, B. (1997) 'Orienting effect of vibrations on interfaces', C.R. Acad. Sci. Paris, Vol. 320, Série IIb, pp. 225-230.

Mullins, W.W. and Sekerka, R.F. (1964) J. Appl. Phys., Vol. 35, p. 444.

Nguyen, T.H., Dabo, Y., Billia, B., Camel, D. and Drevet, B. (2001) 'Solidification dirigée d'alliages Al-3,5 \%pds Ni: analyse des étapes préliminaires de fusion et de stabilisation', J. Phys. IV France, Vol. Il, pp. 161-167.

Nield, D.A. (1964) 'Surface tension and buoyancy effects in cellular convection', J. Fluid Mech., Vol. 19, pp. 341-352. 
Plapp, M. and Karma, A. (1999) 'Eutectic colony formation: n stability analysis', Physical Review E (Statistical Physics, Plasmas, Fluids, and Related interdisciplinary Topics), Vol. 60, pp. 6865-6889.

Plapp. M. and Karma, A. (2001) 'Eutectic co formation: a phase field model', arXiv:condmat/0112194 v1 11 dec, pp. 1-18

Prud'homme, R. and Zappoli, B. (Eds.) (2003) Bilan à quatre ans 2000-2003 du GDR 2258 Phénomènes de Transport et Transitions de Phase en Micropesanteur', Septembre.

Prud'homme, R., Langevin, D. and Faivre, G. (Eds,) (2001) 'Sciences de la Matière et Microgravité, Colloque', J. Phys. IV France, Vol. 86, p. I.

Prud'homme, R. and El Ganaoui, M (Eds) (2004) Microgravity, CR Acad. Sc. Meca. t.332, fasc. 56, Elsevier.

Scriven, LE. and Sternling C.V. (1964) 'On cellular convection driven by surface-tension gradients: effects of mean surface tension and surface viscosity', Chem. Engng. Mech., Vol. 19, pp. 321-340.

Seppecher, P. (1987) Etude d'une modélisation des zones capillaires fluides: interfaces et lignes de contact, $\mathrm{PhD}$ Thesis, University Pierre and Marie Curie, Paris.

Sympson, JE. and Garimella, S.V. (2000) 'The influence of gravity levels on the horizontal Bridgman crystal growth of an alloy', Int. J. Heat Mass Transf. Vol. 43, pp. 1905-1923.

Timchenko, V., Bennacer, R., Leonardi, E. and de Vahl Davis, G. (2002) 'The influence of Soret effect on morphological instability during directional solidification of a binary alloys', in S. Dost, H. Struchrup and I. Dincer (Eds.) Progress in Transport Phenomena, Paris: Elsevier, pp. 6ll-616,

Touhiri, R., Benhadid, H., and Henry, D. (1999) 'Stabilisation par champ magnétique de la convection en cavité cylindrique chauffée par le bas', J. Chim. Phys., Vol. 96, pp. 1098-1104.

\section{Nomenclature}

$\begin{array}{ll}G r & \text { Grashov number } \\ H a & \text { Hartman number } \\ P r & \text { Prandtl number } \\ R a & \text { Rayleigh number } \\ R e & \text { Reynolds number } \\ S c & \text { Schmidt number } \\ \text { Ste } & \text { Stefan number } \\ B & \text { Magnetic field magnitude } \\ c & \text { Specific heat of the material } \\ C & \text { Solute concentration, average mass fraction } \\ \widetilde{C} & \text { Concentration of impurities } \\ D & \text { Mass diffusivity } \\ \mathrm{E} & \text { Internal energy per unit mass } \\ f_{t} & \text { Volume fraction of the liquid } \\ g & \text { Gravitational acceleration } \\ G & \text { Thermal gradient } \\ h & \text { Enthalpy per unit mass, interpolation function } \\ k & \text { Thermal conductivity, segregation coefficient } \\ K & \text { Isotropic permeability } \\ L & \text { Reference length } \\ L_{f} & \text { Latent heat of solidification } \\ m & \text { Liquidus slope }\end{array}$


$M \quad$ Mobility function of the eutectic

$\tilde{M} \quad$ Mobility function of the impurities

$p \quad$ Pressure

$s \quad$ Entropy per unit mass

$t \quad$ Time

$T, T_{f}$ Mixture temperature, melting temperature,

$u \quad x$ velocity component, scaled concentration

$\vec{U} \quad$ Local fluid velocity

$v \quad z$ velocity components

$V, V_{t} \quad$ Melting velocity, pulling velocity

$\vec{v} \quad$ Local velocity

$W \quad$ Characteristic thickness

$x \quad$ Horizontal coordinate

$y, z \quad$ Vertical coordinate

$\beta_{T} \quad$ Thermal expansion coefficient of the liquid

$\Delta \quad$ Jump

$\lambda$ Thermal diffusivity, capillarity coefficient

$\mu, v \quad$ Dynamical viscosity, kinematical viscosity

$\rho \quad$ Density

$\phi \quad$ Phase field

$\sigma \quad$ Electrical conductivity

Subscripts

c Cold

E Eutectic

$F \quad$ Melting

$h \quad$ Hot

$l, s, s l$ Liquid, solid, transition zone

$\alpha, \beta \quad$ Solid phases in a binary eutectic 